\title{
The oculomotor-tentorial triangle. Part 1: microsurgical anatomy and techniques to enhance exposure
}

\author{
Ali Tayebi Meybodi, MD, Sirin Gandhi, MD, Justin Mascitelli, MD, Baran Bozkurt, MD, \\ Gyang Bot, MD, Mark C. Preul, MD, and Michael T. Lawton, MD
}

Department of Neurosurgery, Barrow Neurological Institute, Phoenix, Arizona

\begin{abstract}
OBJECTIVE Access to the ventrolateral pontomesencephalic area may be required for resecting cavernous malformations, performing revascularization of the upper posterior circulation, and treating vascular lesions such as aneurysms. However, such access is challenging because of nearby eloquent structures. Commonly used corridors to this surgical area include the optico-carotid, supracarotid, and carotid-oculomotor triangles. However, the window lateral to the oculomotor nerve can also be used and has not been studied. The authors describe the anatomical window formed between the oculomotor nerve and the medial tentorial edge (the oculomotor-tentorial triangle [OTT]) to the ventrolateral pontomesencephalic area, and assess techniques to expand it.
\end{abstract}

METHODS Four cadaveric heads ( 8 sides) underwent orbitozygomatic craniotomy. The OTT was exposed via a pretemporal approach. The contents of the OTT were determined and their anatomical features were recorded. Also, dimensions of the brainstem surface exposed lateral and inferior to the oculomotor nerve were measured. Measurements were repeated after completing a transcavernous approach (TcA), and after resection of temporal lobe uncus (UnR).

RESULTS The $\mathrm{s}_{1}$ segment and proximal $\mathrm{s}_{2}$ segment of the superior cerebellar artery (SCA) and $\mathrm{P}_{2 \mathrm{~A}}$ segment of the posterior cerebral artery (PCA) were the main contents of the OTT, with average exposed lengths of $6.4 \pm 1.3 \mathrm{~mm}$ and $5.5 \pm 1.6 \mathrm{~mm}$ for the SCA and PCA, respectively. The exposed length of the SCA increased to $9.6 \pm 2.7 \mathrm{~mm}$ after TcA $(p=0.002)$, and reached $11.6 \pm 2.4 \mathrm{~mm}$ following UnR $(p=0.004)$. The exposed PCA length increased to $6.2 \pm 1.6 \mathrm{~mm}$ after TcA $(p=0.04)$, and reached $10.4 \pm 1.8 \mathrm{~mm}$ following UnR $(p<0.001)$. The brainstem surface was exposed $7.1 \pm$ $0.5 \mathrm{~mm}$ inferior and $5.6 \pm 0.9 \mathrm{~mm}$ lateral to the oculomotor nerve initially. The exposure inferior to the oculomotor nerve increased to $9.3 \pm 1.7 \mathrm{~mm}$ after TcA ( $p=0.003)$, and to $9.9 \pm 2.5 \mathrm{~mm}$ after $\operatorname{UnR}(p=0.21)$. The exposure lateral to the oculomotor nerve increased to $8.0 \pm 1.7 \mathrm{~mm}$ after TcA $(p=0.001)$, and to $10.4 \pm 2.4 \mathrm{~mm}$ after $\operatorname{UnR}(p=0.002)$.

CONCLUSIONS The OTT is an anatomical window that provides generous access to the upper ventrolateral pontomesencephalic area, $\mathrm{s}_{1}$ - and $\mathrm{s}_{2}-\mathrm{SCA}$, and $\mathrm{P}_{2 \mathrm{~A}}-\mathrm{PCA}$. This window may be efficiently used to address various pathologies in the region and is considerably expandable by TcA and/or UnR.

https://thejns.org/doi/abs/10.3171/2018.1.JNS173139

KEYWORDS ambient cistern; crural cistern; orbitozygomatic approach; pontomesencephalic junction; posterior circulation revascularization; pretemporal approach; subtemporal approach; transcavernous approach; vascular disorders; anatomy

A CCESS to the ventrolateral aspect of the pontomesencephalic area and adjacent cisterns may be required to address a variety of pathologies. These pathologies include but are not limited to brainstem gliomas, cavernous malformations, and vascular lesions such as aneurysms..$^{5,11,13,35,39}$ However, such access is mainly limited due to the abundance of eloquent structures residing within the anterior and middle incisural spaces. ${ }^{27}$ Two approaches are commonly used for accessing this region: 1) transsylvian and 2) subtemporal approaches. $3.22,28,33,34$, 38,45 The transsylvian approach mainly enables access to the interpeduncular and upper prepontine cisterns, while

ABBREVIATIONS $\mathrm{AChA}=$ anterior choroidal artery; $\mathrm{AMZ}$ = anterior mesencephalic zone; $\mathrm{BA}=$ basilar artery; $\mathrm{OTT}=$ oculomotor-tentorial triangle; $\mathrm{PCA}=$ posterior cerebral artery; $\mathrm{PCOA}=$ posterior communicating artery; $\mathrm{PCP}=$ posterior clinoid process; $\mathrm{PMS}=$ pontomesencephalic sulcus; $\mathrm{SCA}=$ superior cerebellar artery; $\mathrm{STZ}=$ supratrigeminal zone; TcA = transcavernous approach; UnR = resection of temporal lobe uncus.

SUBMITTED December 14, 2017. ACCEPTED January 15, 2018.

INCLUDE WHEN CITING Published online June 29, 2018; DOI: 10.3171/2018.1.JNS173139. 


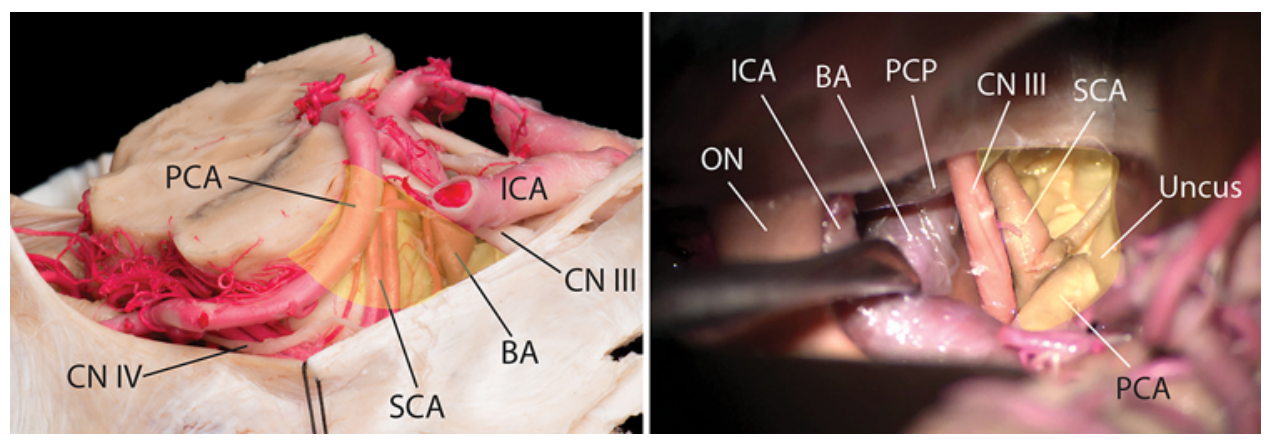

FIG. 1. Left: Cadaveric dissection showing the right tentorial incisura from a lateral perspective. The OTT (yellow shaded area) is formed between the cisternal segment of the oculomotor nerve medially, and the free edge of the tentorium laterally. The PCA and SCA are the main vascular contents of the triangle, although the BA may also be accessed through the OTT. Right: Exposure of the right OTT in another specimen after completing an orbitozygomatic craniotomy and developing a pretemporal corridor. The posterior aspect of the OTT (yellow shaded area) is delimited by the cerebral peduncle and PCA coursing around it medially, and uncus of the temporal lobe laterally. $\mathrm{CN}=$ cranial nerve; $\mathrm{ICA}=$ internal carotid artery; $\mathrm{ON}=$ optic nerve; $\mathrm{PCP}=$ posterior clinoid process. Copyright Michael T. Lawton. Published with permission. Figure is available in color online only.

the subtemporal approach grants access to the ambient cistern. ${ }^{38}$ However, access to the crural cistern that lies between the interpeduncular and ambient cisterns is limited in both approaches..$^{17,25,38}$ In a transsylvian approach, as the surgical target moves posterolaterally around the cerebral peduncle, the temporal lobe and the tentorium become major obstacles to more lateral and posterior exposure. ${ }^{1,23,37,38}$ Likewise, moving anteriorly from the ambient cistern toward the crural and interpeduncular cisterns during a subtemporal exposure, the surgical maneuverability becomes progressively limited as the target assumes a deeper position on the ventral aspect of the midbrain. ${ }^{38}$

Triangular corridors are defined to facilitate orientation during these surgical approaches. The most well-known and studied of these corridors are the supracarotid, carotidoculomotor, and optico-carotid triangles. ${ }^{9,19,20,42}$ However, these triangles are mainly used to access the interpeduncular cistern and its contents, including the basilar apex. On the other hand, the triangular corridor formed between the oculomotor nerve and medial tentorial edge (Fig. 1), which we call the oculomotor-tentorial triangle (OTT), has not been studied despite its frequent use to address various pathologies. ${ }^{11,16,36,43}$ The purpose of this two-part report is to define the microsurgical anatomy of the OTT and assess ways to expand it (Part 1), and to summarize our clinical experience using the OTT to address various cerebrovascular pathologies, including aneurysms, cavernous malformations, and dural arteriovenous fistulas (Part 2).

\section{Methods}

\section{Pretemporal Approach and Exposure of the OTT}

Four cadaveric heads embalmed in a customized alcohol-based solution were infused with colored silicone into arteries (red) and veins (blue), and prepared for dissection. Each head was placed in a 3-pin Mayfield clamp (Integra) and turned $30^{\circ}$ to the contralateral side. The vertex was slightly tilted toward the floor such that the malar eminence was the highest point in the surgical field. A large question mark-shaped incision was started just anterior to the tragus, curved around the pinna, and then anterosuperiorly to reach the midline just behind the hairline. After reflection of the musculocutaneous flap using a subfascial technique, a large two-piece pterional-orbitozygomatic craniotomy was performed. The dura was opened with a curvilinear incision, and a complete sylvian split was performed using a surgical microscope (Carl Zeiss). The carotid, interpeduncular, chiasmatic, and crural cisterns were opened to gain entry to the anterior incisural space. Next, a pretemporal approach was performed as described by de Oliveira et al. ${ }^{8}$ To perform this approach, the venous attachments between the tip of the temporal lobe and the sphenoparietal sinus were divided. Also, the following surgical maneuvers were performed to maximize posterior mobilization of the temporal lobe: 1) detachment of arachnoid adhesions between the medial aspect of the temporal lobe, the oculomotor nerve, and the cisternal course of the anterior choroidal artery (AChA); and 2) releasing the cisternal segment of the anterior temporal artery from arachnoid attachments. ${ }^{35}$ Using a table-mounted brain retractor system (Integra), we retracted the temporal lobe posteriorly. The frontal lobe was also retracted by placing the retractor blade on the orbital gyri lateral to the olfactory tract. Retraction forces were adjusted to maximize retraction while avoiding any gross injury to the underlying neural tissue. The retractors were then fixed and locked in place.

\section{Contents and Morphometrics of the OTT}

The boundaries and contents of the OTT were defined. Next, the following measurements were recorded using the Treon Plus frameless stereotactic navigation system (Medtronic): 1) length of the exposed oculomotor nerve, 2) length of the exposed tentorium starting from its crossing point with the oculomotor nerve at the oculomotor trigone, 3 ) length of the exposed superior cerebellar artery (SCA) and 4) posterior cerebral artery (PCA), and 5) length of exposed basilar artery (BA) through the OTT. Using the stereotactic navigation system, we recorded the spatial coordinates of the exposed OTT vertices to calculate its surface area.

Additionally, to assess the exposed surface of the anterolateral pontomesencephalic area, the following points were marked: 1) the point of emergence of the oculomotor 
nerve just medial to the cerebral peduncle (Fig. 2 point A), 2) the inferior-most exposed point on the brainstem surface along a vertical line passing through point A (Fig. 2 point $\mathrm{B}), 3$ ) the lateral-most exposed point on the cerebral peduncle at the axial level of point A (Fig. 2 point $C$ ), and 4) the most inferior exposed point on the brainstem surface along a vertical line passing through point $C$ (Fig. 2 point $\mathrm{D}$ ). The lengths of lines $\mathrm{A}-\mathrm{B}, \mathrm{A}-\mathrm{C}$, and $\mathrm{C}-\mathrm{D}$ were measured using the stereotactic navigation system.

\section{Expanding the OTT}

Keeping the brain retractors fixed in place, two skull base approaches were then performed consecutively to evaluate techniques to enhance the exposure gained through the OTT. First, a transcavernous approach (TcA) was performed by the following steps to increase the mobility of the third cranial nerve:2,31 1 ) intradural anterior clinoidectomy; 2) opening the roof of the cavernous sinus medial to the oculomotor nerve to release the cavernous segment of the oculomotor nerve and anterior genu of the cavernous carotid artery; and 3) posterior clinoidectomy. The above-mentioned measurements were repeated (except the length of the exposed tentorium and oculomotor nerve) after TcA. Next, the temporal lobe uncus was resected (UnR) as described by Post et al., ${ }^{24}$ and the variables were measured again (Fig. 3).

\section{Statistical Analysis}

IBM SPSS statistical software (version 24.0, IBM Corp.) was used to perform statistical analysis. The paired t-test was used to compare dependent means of parametric variables between the initial exposure and TcA, and between the TcA and UnR steps.

\section{Results}

\section{Pretemporal Transsylvian Approach}

The OTT was successfully exposed in all specimens. It was formed between the following structures: 1) the oculomotor nerve anteromedially, 2) the medial tentorial edge anterolaterally, 3) the cerebral peduncle posteromedially, and 4) the temporal lobe uncus posterolaterally (Figs. 1, $3 \mathrm{~A}$, and $3 \mathrm{~B})$. It enabled exposure of the crural cistern just lateral to the oculomotor nerve. The pretemporal transsylvian approach enabled exposure of the full cisternal length of the oculomotor nerve from its origin to the oculomotor trigone at the roof of the cavernous sinus (average 16.5 $\pm 2.8 \mathrm{~mm}$ ). The tentorial edge was exposed at an average length of $10.1 \pm 1.5 \mathrm{~mm}$ (Fig. 3). The entry point of the trochlear nerve to the tentorium was not visible in any of the specimens after the pretemporal approach.

\section{Vessel Exposure}

The $\mathrm{s}_{1}$ segment of the SCA, proximal $\mathrm{s}_{2}$ segment of the SCA, and $\mathrm{P}_{2 \mathrm{~A}}$ segment of the PCA were the main contents of the OTT in all specimens. The origin of the SCA was visible through the OTT in all specimens. The $\mathrm{P}_{2 \mathrm{P}}$ segment of the PCA was not visualized via the OTT in any of the specimens. The average lengths of the exposed PCA and SCA were $5.5 \pm 1.6 \mathrm{~mm}$ and $6.4 \pm 1.3 \mathrm{~mm}$, respectively.

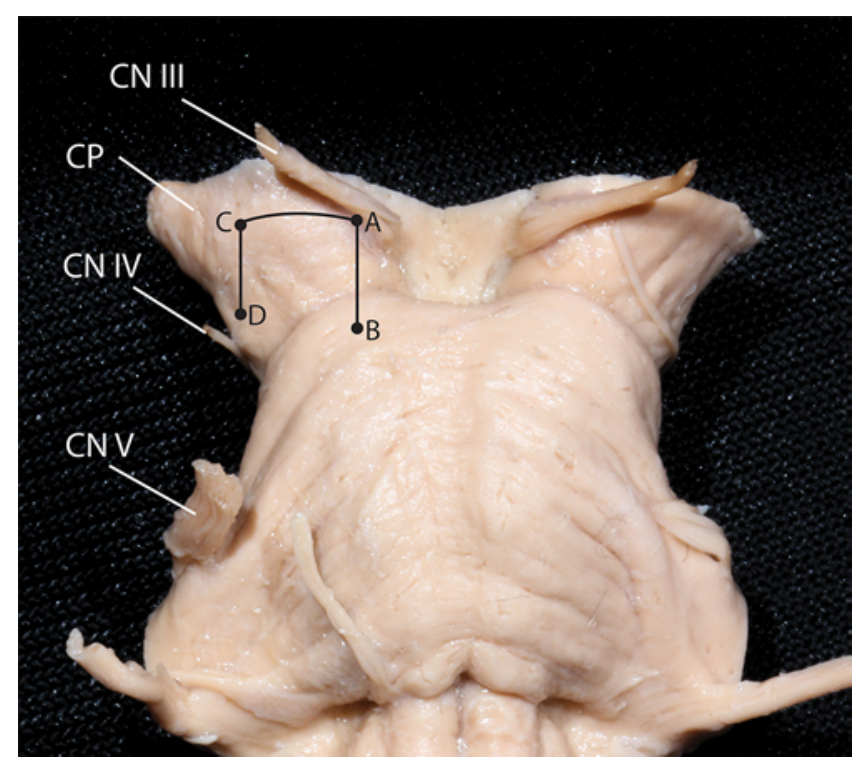

FIG. 2. Depiction of the measurement of the dimensions of the ventrolateral pontomesencephalic area through the OTT. Point A is at the root exit zone of the oculomotor nerve just medial to the cerebral peduncle (CP); point $B$ is the inferior-most exposed point on the brainstem surface along a vertical line passing through point $A$; point $C$ is the lateral-most exposed point on the cerebral peduncle at the axial level of point $A$; point $D$ is the inferior-most exposed point on brainstem surface along a vertical line passing through point $C$. The lengths of lines $A-B, A-C$, and C-D were calculated. Copyright Michael T. Lawton. Published with permission. Figure is available in color online only.

The basilar apex was not visible through the OTT in any of the specimens. On the other hand, the basilar trunk was exposed below the level of the SCA origin in all specimens. The main anatomical obstacles to visualize the basilar trunk through the OTT were the limited medial mobilization of the oculomotor nerve and the posterior clinoid process (PCP) lying just medial to the oculomotor nerve. The PCP and third cranial nerve were the main obstacles to visualize the proximal segment of the $\mathrm{s}_{1}$-SCA, whereas the tentorial edge mainly hindered exposure of the distal segment of the $\mathrm{s}_{2}-\mathrm{SCA}$. In all specimens, the $\mathrm{P}_{1}-\mathrm{P}_{2}$ junction was medial to, or just on top of, the oculomotor nerve (Fig. 3 ). There was no anatomical structure limiting exposure of the proximal $\mathrm{P}_{2 \mathrm{~A}}-\mathrm{PCA}$. However, the temporal lobe was the main limiting factor for exposing more distal segments of the PCA (i.e., distal $\mathrm{P}_{2 \mathrm{~A}}$ and $\mathrm{P}_{2 \mathrm{P}}$ segments).

\section{Brainstem Exposure}

Average cerebral peduncle width exposed through the OTT at the level of the oculomotor nerve (line A-C in Fig. 2) was $5.6 \pm 0.9 \mathrm{~mm}$. Average height of the exposed brainstem along the sagittal plane of the oculomotor nerve root emergence point just medial to the cerebral peduncle (line A-B in Fig. 2) was $7.1 \pm 0.5 \mathrm{~mm}$. This exposure extended inferior to the pontomesencephalic sulcus (PMS) in all specimens (Fig. 4). An average of $5.0 \pm 1.3 \mathrm{~mm}$ of the brainstem height was exposed inferior to the lateralmost exposed point on the cerebral peduncle (line C-D in Fig. 2). The results of measurements of the OTT during the pretemporal approach and following $\mathrm{TcA}$ and UnR are listed in Tables 1 and 2. 
Tayebi Meybodi et al.
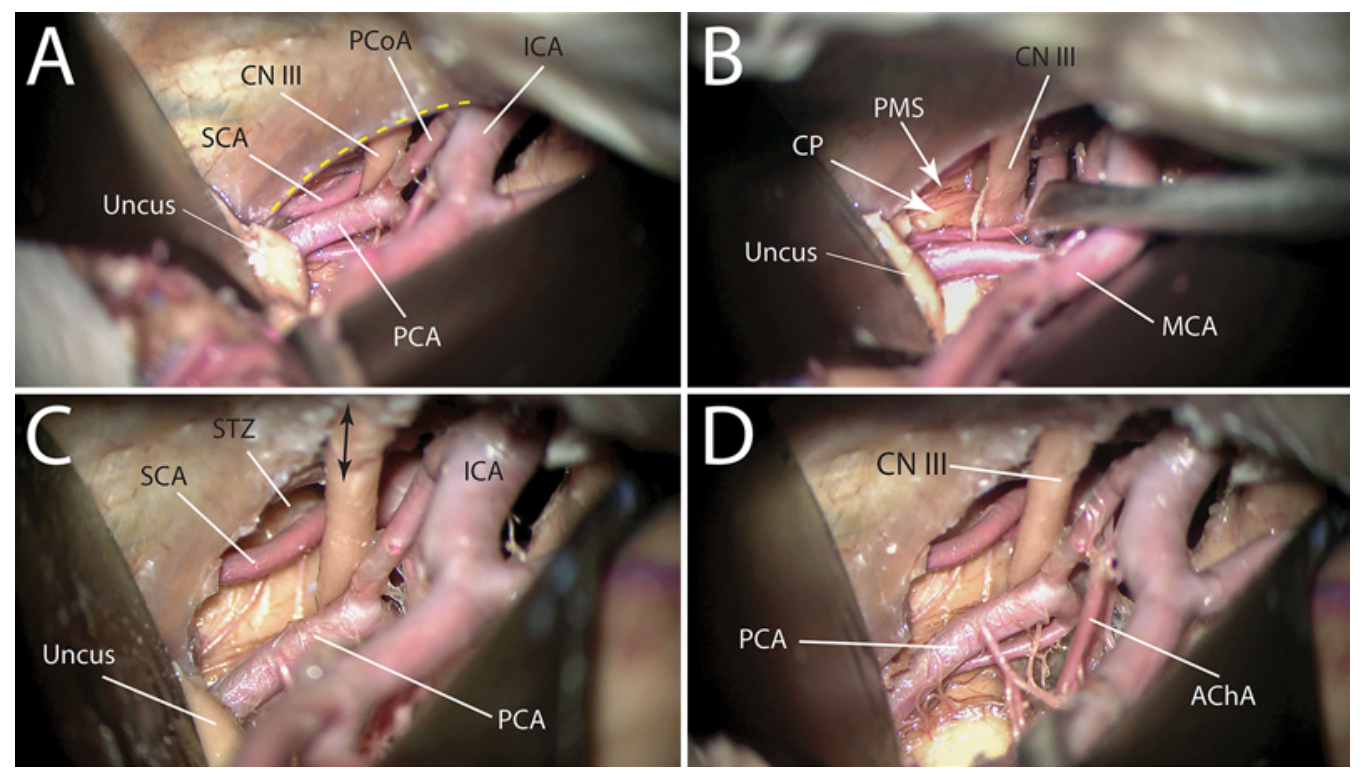

FIG. 3. Exposure and stepwise enhancement of the left OTT. A: The OTT is exposed after completing a pretemporal approach. The $s_{1}$ - and proximal $s_{2}-S C A$ and $P_{2 A}-P C A$ are exposed via the OTT. The dashed line designates the medial tentorial edge. $B$ : The PMS is also exposed through the OTT after slight manipulation of the SCA and PCA. Note that the tentorial edge and temporal uncus are the major obstacles to exposing further posterior segments of the SCA and PCA toward the ambient cistern. The tentorium also blocks exposure of the brainstem surface below the level of PMS. C: After completion of a TcA, the cavernous segment of the oculomotor nerve is exposed (double-arrow line), which enables further mobilization. This maneuver enables better exposure of the STZ that is located inferior to the PMS at the sagittal level of the root exit zone of the oculomotor nerve. Further posterior segments of the SCA and PCA are exposed as well. However, the temporal uncus is still a major obstacle in exposing the SCA and PCA toward the ambient cistern. D: Resection of the uncus enables more distal segments of the $s_{2}-S C A$ and $P_{2 A}-P C A$. MCA $=$ middle cerebral artery. Copyright Michael T. Lawton. Published with permission. Figure is available in color online only.

\section{Transcavernous Approach}

TcA led to an increase in the exposed length of the oculomotor nerve anteriorly extending to the superior orbital fissure (Fig. 3C). This caused doubling of the surface area of the OTT (Fig. 5). Completing a posterior clinoid- ectomy following the anterior clinoidectomy and opening of the cavernous sinus roof increased the mobility of the oculomotor nerve. Subsequently, the exposed lengths of all exposed vessels increased, which was statistically significant (Table 1, Fig. 5). Also, all dimensions of the exposed surface of the ventrolateral pontomesencephalic area in-
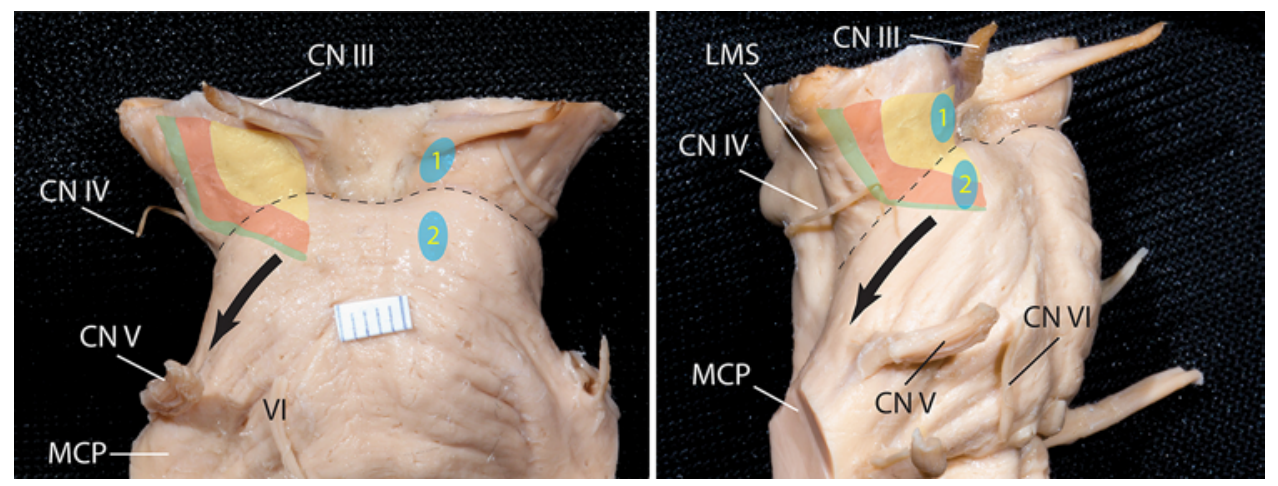

FIG. 4. Depictions of exposed surface of the brainstem on a cadaveric specimen through the OTT with a transsylvian pretemporal approach (yellow shaded area), and its enhancement following a TcA (red shaded area) and resection of the temporal uncus (green shaded area). Anterior (left) and anterolateral (right) perspectives are shown. Areas 1 and 2 designate the anterior mesencephalic and supratrigeminal safe entry zones of the brainstem. It is evident that the AMZ is sufficiently exposed using the transsylvian pretemporal approach, whereas optimal exposure of the STZ may require expanding the OTT with a TcA. The resection of the temporal uncus after the TcA enables further posterior exposure of the ventrolateral cerebral peduncle, but does not extend to the lateral mesencephalic sulcus (LMS) that is typically exposed during a subtemporal approach. The dashed line shows the PMS. The black arrow represents the course of the pontocerebellar (transverse pontine) fibers that pass through the middle cerebellar peduncle (MCP) to reach the cerebellum. Copyright Michael T. Lawton. Published with permission. Figure is available in color online only. 
TABLE 1. Results of measurement of exposed lengths of PCA, SCA, and BA through the OTT after TS, TS + TCA, and TS + TCA + UnR approaches and their statistical comparison

\begin{tabular}{lccccc}
\hline Artery & TS & TS + TcA & p Value* & TS + TcA + UnR & $p$ Value† \\
\hline BA & $4.8 \pm 1.1$ & $7.2 \pm 1.2$ & $<0.001$ & $8.1 \pm 1.9$ & 0.07 \\
\hline SCA & $6.4 \pm 1.3$ & $9.6 \pm 2.7$ & 0.002 & $11.6 \pm 2.4$ & 0.004 \\
\hline PCA & $5.5 \pm 1.6$ & $6.2 \pm 1.6$ & 0.04 & $10.4 \pm 1.8$ & $<0.001$ \\
\hline
\end{tabular}

TS = Transsylvian pretemporal.

Measurements given as means \pm standard deviations $(\mathrm{mm})$.

* Result of paired t-test to compare TS with TS + TcA.

† Result of paired t-test to compare TS + TcA with TS + TcA + UnR

creased significantly after TcA ( $\mathrm{p} \leq 0.01$; Table 2 , Figs. 4 and 6).

\section{Uncal Resection}

Resection of the temporal lobe uncus allowed further exposure of the tentorium posteriorly. Following UnR, the surface area of the OTT showed a 30\% increase with a statistically significant difference compared with the surface area exposed after TcA ( $p=0.02$; Fig. 5). Furthermore, compared to the TcA, it led to a statistically significant increase in the exposed lengths of the PCA and SCA, but not the BA (Table 1, Fig. 5). The width of the cerebral peduncle (line $\mathrm{A}-\mathrm{C}$ in Fig. 2) was the only parameter that showed a statistically significant increase after UnR (Table 2, Figs. 4 and 6). Figure 4 depicts the approximate surface of the ventrolateral pontomesencephalic area after performing the three approaches. UnR enabled exposure of the entry point of the trochlear nerve to the tentorium (porus trochlearis) in 50\% (4/8) of specimens.

\section{Discussion}

Our results show that the OTT is an efficient corridor to expose the anterior and proximal lateral perimesencephalic segments of the PCA and SCA, and the upper basilar trunk below the level of SCA origin. It also provides a generous exposure of the ventrolateral pontomesencephalic area that is more expanded after performing TcA and UnR (Table 2, Figs. 4 and 6). Therefore, this corridor could be efficiently used to expose these anatomical structures/regions in the ventrolateral pontomesencephalic area for treatment of various pathologic states.

\section{Exposure of Arterial Branches}

\section{Basilar Apex and Basilar Trunk}

The OTT enabled the exposure of the basilar trunk below the level of the SCA in all specimens; however, the basilar apex was not visible through the OTT. These results are of surgical significance. First, when the basilar apex aneurysms are exposed through a transsylvian approach, the temporary clipping of the basilar trunk could be performed through the OTT or the carotid-oculomotor triangle. Placing a proximal BA clip through the OTT would allow the surgeon to have more room while working on the aneurysm through the carotid-oculomotor and optico-carotid triangles. Furthermore, when the basilar apex is low-
TABLE 2. Results of measurement of exposed dimensions of the brainstem through the OTT after TS, TS + TcA, and TS + TcA + UnR approaches and their statistical comparison

\begin{tabular}{|c|c|c|c|c|c|}
\hline Dimension & TS & $\mathrm{TS}+\mathrm{TcA}$ & $\begin{array}{c}p \\
\text { Value* }\end{array}$ & $T S+T c A+U n R$ & $\begin{array}{c}p \\
\text { Value† }\end{array}$ \\
\hline Line $A-B$ & $7.1 \pm 0.5$ & $9.3 \pm 1.7$ & 0.003 & $9.9 \pm 2.5$ & 0.21 \\
\hline Line A-C & $5.6 \pm 0.9$ & $8.0 \pm 1.7$ & 0.001 & $10.4 \pm 2.4$ & 0.002 \\
\hline Line C-D & $5.0 \pm 1.2$ & $7.7 \pm 2.4$ & 0.01 & $8.1 \pm 2.1$ & 0.34 \\
\hline
\end{tabular}

Measurements given as means \pm standard deviations $(\mathrm{mm})$.

* Result of paired t-test to compare TS with TS + TcA.

† Result of paired t-test to compare TS + TcA with TS + TcA + UnR.

lying, exposure and clipping of the basilar apex aneurysm may be better performed through the OTT, which provides a more horizontal view across the basilar apex compared to the optico-carotid and carotid-oculomotor triangles that provide a more vertical view. ${ }^{19}$

The main obstacles to exposing greater lengths of the BA through the OTT were the PCP and limited mobility of the oculomotor nerve. The TcA significantly increased the exposed length of the basilar trunk through the OTT as it eliminates the PCP and increases the mobility of the oculomotor nerve. ${ }^{2}$ Overall, TcA could be efficiently used to increase the working area on the basilar apex and trunk $\mathrm{k}^{4,10}$ while simultaneously providing more room to place the proximal basilar clip through the OTT.

\section{SCA and PCA}

The OTT enables exposing the $\mathrm{P}_{2 \mathrm{~A}}-\mathrm{PCA}$, and $\mathrm{s}_{1}-$ and proximal $\mathrm{s}_{2}-\mathrm{SCA}$. Although rare, aneurysms of the $\mathrm{P}_{2 \mathrm{~A}^{-}}$ PCA may be efficiently exposed through the OTT. ${ }^{11,23,30,39}$ Additionally, when the SCA or PCA is targeted as a recipient in cerebral revascularization procedures, the OTT enables exposure of generous lengths of these arteries (Figs. 3 and 5). ${ }^{21,29,35,43}$ The use of OTT to perform a bypass procedure on the SCA or PCA has several advantages. First, the crural cistern is a more spacious area with less surgical depth than the interpeduncular cistern. Therefore, performing a bypass would be technically less demanding compared to the PCA/SCA targeted in the interpeduncular cistern. Second, the lengths of the recipient SCA and PCA may be greater than those exposed in the interpeduncular cistern. ${ }^{36}$ Third, as a bypass requires temporary trapping of the recipient artery, such a maneuver would endanger thalamoperforating arteries when performed in the interpeduncular cistern. On the other hand, the risk to thalamoperforating arteries is less during clipping of $\mathrm{P}_{2 \mathrm{~A}^{-}}$ PCA because it harbors fewer perforating arteries to the thalamus, although peduncular perforating and thalamogeniculate arteries may still be at risk. ${ }^{26,44}$

The oculomotor nerve and PCP were the main obstacles to exposing greater lengths of proximal SCA, whereas the tentorial edge was the main limiting structure to expose distal segments of the SCA. TcA requires resection of the PCP and increases mobilization of the oculomotor nerve and the tentorial edge (Fig. 3). ${ }^{2,31}$ This maneuver could therefore create more room for performing a bypass on the SCA. TcA, however, did not expose a significantly greater length of the PCA (Fig. 5). This is a predictable 

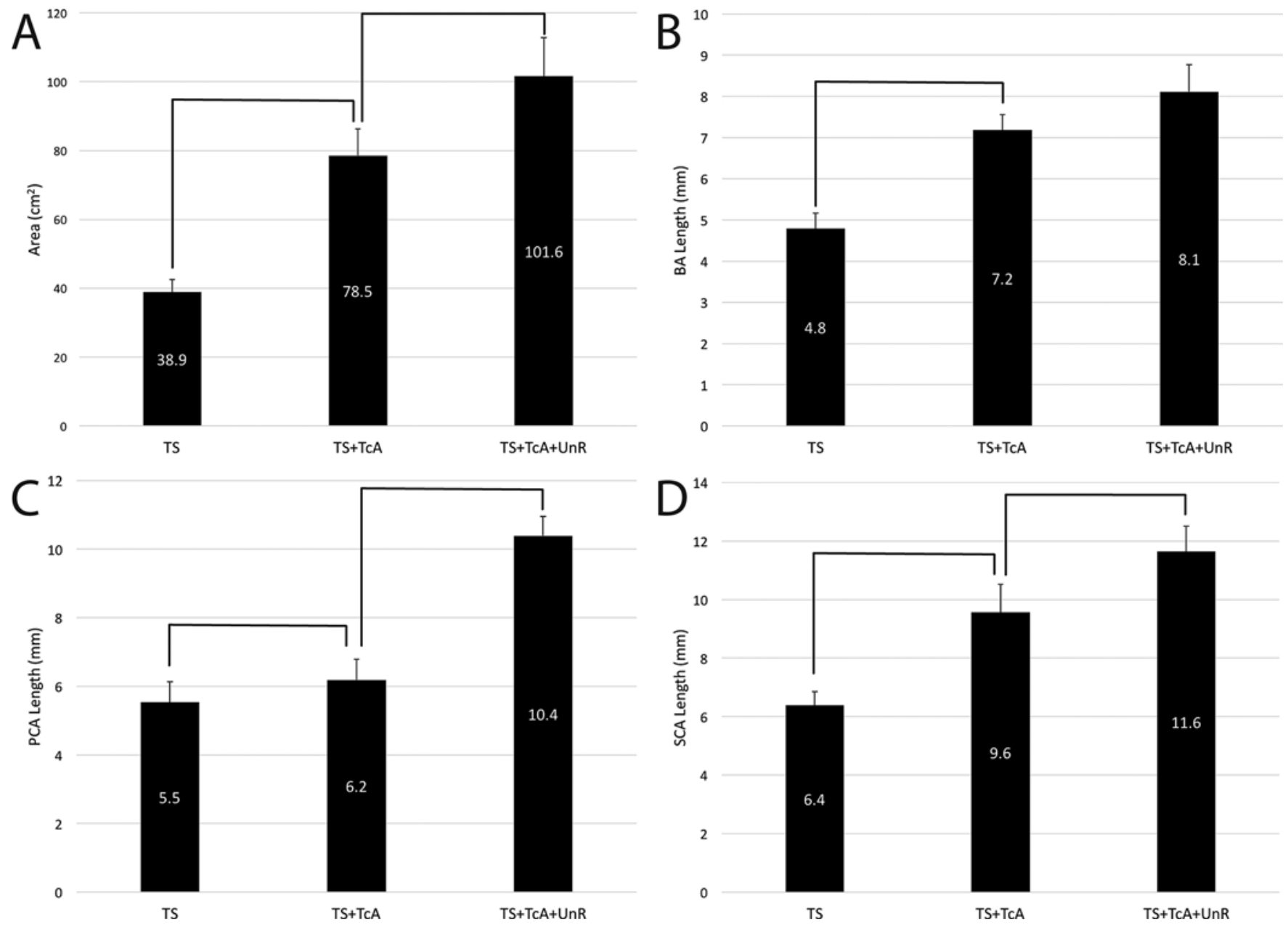

FIG. 5. Graph representations of the area of the OTT (A), and exposed lengths of the BA (B), PCA (C), and SCA (D) through the OTT during a transsylvian pretemporal (TS) approach, and following completion of the TcA and UnR. Brackets show statistically significant increases (paired t-test).

result because the PCA courses above the tentorial edge and the oculomotor nerve, whereas the SCA courses underneath the tentorial edge and the third cranial nerve.

UnR led to an increase in exposed lengths of both the SCA and PCA (Fig. 5). However, the increase in exposed PCA length was more significant. This could be explained by the anatomical position of the $\mathrm{P}_{2 \mathrm{~A}}-\mathrm{PCA}$ medial to the temporal lobe uncus (which forms the anterolateral limit of the crural cistern), ${ }^{27}$ whereas the SCA eventually courses below the tentorial edge in the cerebellomesencephalic fissure.

The OTT was not a favorable window to expose the entry point of the trochlear nerve to the tentorium, although TcA + UnR enabled its visualization in 50\% (4/8) of specimens. This finding shows that the OTT may, at most, expose the most anterior parts of the ambient cistern and is not an optimal window to expose lesions located more posteriorly (e.g., $\mathrm{P}_{2 \mathrm{P}}$-PCA). This finding is in concordance with previous studies emphasizing that the $\mathrm{P}_{2 \mathrm{P}}$-PCA could not be exposed during the pretemporal approach.,11,37,38

\section{Alternative Approaches}

The exposure of basilar trunk, $\mathrm{P}_{2 \mathrm{~A}}-\mathrm{PCA}$, and $\mathrm{s}_{2}-\mathrm{SCA}$ may be obtained via a subtemporal approach with or without anterior petrosectomy. ${ }^{12,23,27,38,39}$ Basically, the subtemporal approach to these arterial segments uses the window between the oculomotor nerve and the tentorium, which is more extended posteriorly along the ambient cistern. While the subtemporal approach provides the advantage of exposing more posterior segments of the PCA and SCA, it has certain limitations. ${ }^{38}$ Temporal lobe retraction is a major drawback of the subtemporal approach that may lead to serious postoperative temporal lobe swelling. ${ }^{32,35,37}$ Furthermore, it should be noted that, compared to the transsylvian approach, the risk of injury to the oculomotor and trochlear nerves is greater during the subtemporal approach. ${ }^{12}$ On the other hand, the temporal lobe is much more tolerant to posterior retraction than to superior retraction. ${ }^{43}$

The transtemporal-transchoroidal and transinsulartranschoroidal approaches enable access to the ambient cistern. ${ }^{14,17,40}$ Compared with the OTT, these approaches provide a better exposure of the posterior parts of the ambient cistern (e.g., $\mathrm{P}_{2 \mathrm{P}}$-PCA) with minimal temporal lobe retraction. However, violation of cortical tissue, and the risk of damage to the hippocampus, thalamus, and 
perforating branches of the AChA, are among the disadvantages. Moreover, exposure of the crural cistern and its contents (e.g., $\mathrm{P}_{2 \mathrm{~A}}-\mathrm{PCA}$ ) is more difficult, requiring dissection through the amygdala and significant retraction on the hippocampal formation. ${ }^{17}$ Therefore, the transchoroidal approaches may be an inferior alternative compared to the OTT when a pathology of the crural and anterior ambient cistern is targeted.

\section{Exposure of the Ventrolateral Pontomesencephalic Area}

The perioculomotor area (i.e., anterior mesencephalic zone [AMZ]) is an area of the ventral mesencephalon just lateral to the root exit zone of the oculomotor nerve in the cerebral peduncle that contains frontopontine fibers (the medial one-fifth of the cerebral peduncle width; Fig. 4). $5,6,18,41$ This area has been defined as a safe entry zone for resection of intrinsic midbrain lesions such as cavernous malformations that do not reach the pial surface. An incision made between the SCA and PCA in this area does not cause neurological deficits. ${ }^{5}$ Figure 4 shows the area of the $\mathrm{AMZ}$ and the areas of the ventrolateral brainstem exposed through the OTT and its expansions following TcA and UnR. It is evident that the OTT is a favorable window to reach the AMZ for resection of intraaxial lesions.

The OTT also enables exposure of the intrinsic lesions of the ventrolateral rostral pons through a safe entry zone called the supratrigeminal zone (STZ), ${ }^{6,15,18}$ This area may be accessed via a vertical incision made inferior to the PMS at the sagittal level of the oculomotor nerve. ' Our results show that TcA may be necessary to optimally expose the STZ through the OTT (Fig. 4). The STZ is an area of ventrolateral pons rostromedial to the root entry zone of the trigeminal nerve that harbors the pontocerebellar fibers coursing through the middle cerebellar peduncle. ${ }^{6,15}$ Hebb and Spetzler used the lateral transpeduncular approach through retrosigmoid craniotomy to reach the STZ. ${ }^{15}$ However, as described and illustrated by Hebb and Spetzler, their approach assumes a tangential trajectory along the brainstem to the ventrolateral pons. Our results show that the STZ could also be exposed through the OTT with a vertical trajectory. This may be an advantage of approaching the STZ using the OTT compared to the lateral transpeduncular approach.

While more lateral and inferior exposure of the ventrolateral pontomesencephalic area may exceed the limits of the AMZ and STZ, these extensions may be used to resect intrinsic brainstem lesions with pial presentation at these exposed areas. Figure 4 shows that TcA is efficient in enhancing exposure of the ventrolateral pontomesencephalic area both inferiorly and laterally, whereas the addition of UnR is mainly advantageous in providing more posterolateral exposure. Nevertheless, the appropriate approach and trajectory should be tailored to the individual case. Meticulous review of the preoperative images and the use of intraoperative image guidance greatly help in achieving this target.

\section{Limitations}

While providing favorable exposure of the anterolateral aspect of the pontomesencephalic area and adjacent vessels, using the OTT as a surgical window may entail some
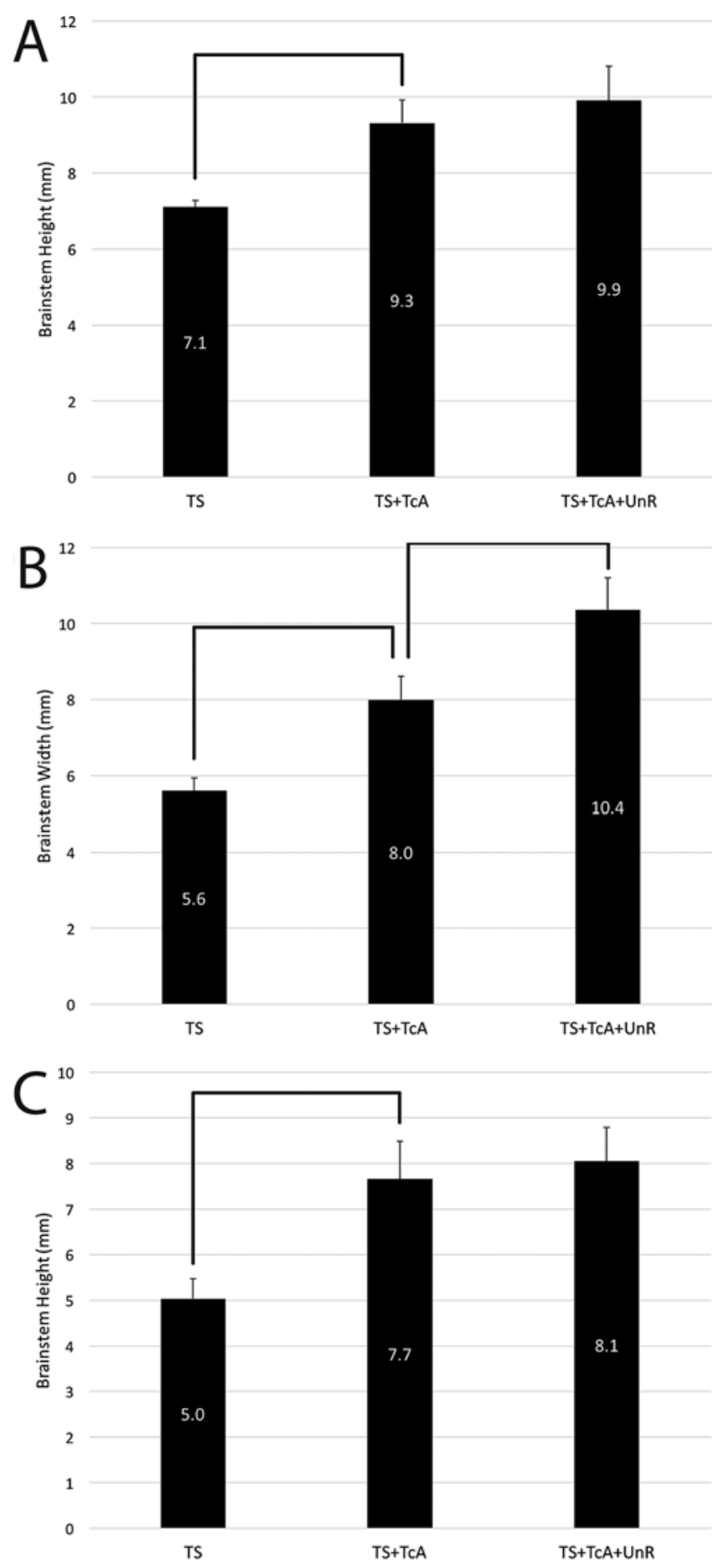

FIG. 6. Graph representations of the exposed dimensions of the brainstem through the OTT during a TS approach, and following completion of the TcA and UnR. A: Height of the brainstem exposed at the sagittal level of the root exit zone of the oculomotor nerve (line A-B, Fig. 2). B: Width of the cerebral peduncle at the axial level of the root exit zone of the oculomotor nerve (line A-C, Fig. 2). C: Height of the brainstem exposed at the sagittal level of the lateral-most exposed point of the width of the cerebral peduncle (line C-D, Fig. 2). Brackets show statistically significant increases (paired t-test). 
challenges and complications. First, it should be noted that posterior retraction of the temporal lobe may also cause problematic postoperative edema. This may be aggravated by severing the bridging veins at the temporal tip draining into the sphenoparietal sinus. Mobilizing the sphenoparietal sinus may obviate the need for division of temporal tip bridging veins. ${ }^{2}$ Second, the oculomotor nerve is inherently at risk when the OTT is used. The risk of oculomotor nerve palsy is further increased during a TcA, although the majority of oculomotor palsies after TcA are reported to be transient. ${ }^{2}$ Furthermore, TcA may injure other contents of the cavernous sinus, especially the trochlear nerve. Third, as exposure of the brainstem surface through the OTT might require manipulation and retraction of the internal carotid and posterior communicating arteries (PCoAs; Fig. 3B), a short fetal-type PCoA may have little mobility and may limit OTT exposure. Fourth, the posterior choroidal branches of the PCA may originate within the OTT. Therefore, care should be taken to identify these branches and protect them accordingly. Finally, the AChA is at risk when the OTT is being exposed (Fig. 3D). As stated above, the internal carotid artery may need some medial retraction for optimal OTT exposure, which mobilizes the AChA. Also, UnR may endanger the AChA coursing medial to the temporal uncus.

\section{Conclusions}

The OTT is an efficient corridor to expose the ventrolateral aspect of the mesencephalon, rostral pons, and adjacent segments of the PCA and SCA. It could be exposed efficiently through a transsylvian pretemporal corridor and expanded with TcA and UnR approaches. This window may be used to treat a variety of neural and vascular pathologies, such as aneurysms and intrinsic midbrain lesions. Also, the exposure of $\mathrm{P}_{2 \mathrm{~A}}-\mathrm{PCA}, \mathrm{s}_{1}-\mathrm{SCA}$, and proximal $\mathrm{s}_{2}-\mathrm{SCA}$ through this triangle could be helpful when these segments of these arteries are targeted for a bypass procedure.

\section{Acknowledgments}

We are grateful to the Barrow Neurological Foundation and to the Newsome Chair in Neurosurgery Research held by Dr. Preul for funding for this project.

\section{References}

1. Aguiar P, Gatto L, Neves M, Martins C, Nakasone F, Isolan G: Aneurysms of the P2P segment of posterior cerebral artery: case report and surgical steps. Case Rep Med 2014:325414, 2014

2. Basma J, Ryttlefors M, Latini F, Pravdenkova S, Krisht A: Mobilization of the transcavernous oculomotor nerve during basilar aneurysm surgery: biomechanical bases for better outcome. Neurosurgery 10 (Suppl 1):106-115, 2014

3. Behari S, Das RK, Jaiswal AK, Jain VK: Fronto-temporoorbitozygomatic craniotomy and "half-and-half" approach for basilar apex aneurysms. Neurol India 57:438-446, 2009

4. Bendok BR, Getch CC, Parkinson R, O'Shaughnessy BA, Batjer HH: Extended lateral transsylvian approach for basilar bifurcation aneurysms. Neurosurgery 55:174-178, 2004

5. Bricolo A: Surgical management of intrinsic brain stem gliomas. Oper Tech Neurosurg 3:137-154, 2000
6. Cavalcanti DD, Preul MC, Kalani MY, Spetzler RF: Microsurgical anatomy of safe entry zones to the brainstem. J Neurosurg 124:1359-1376, 2016

7. Cavalheiro S, Yagmurlu K, da Costa MD, Nicácio JM, Rodrigues TP, Chaddad-Neto F, et al: Surgical approaches for brainstem tumors in pediatric patients. Childs Nerv Syst 31:1815-1840, 2015

8. de Oliveira E, Tedeschi H, Siqueira MG, Peace DA: The pretemporal approach to the interpeduncular and petroclival regions. Acta Neurochir (Wien) 136:204-211, 1995

9. Evans JJ, Hwang YS, Lee JH: Pre- versus post-anterior clinoidectomy measurements of the optic nerve, internal carotid artery, and opticocarotid triangle: a cadaveric morphometric study. Neurosurgery 46:1018-1023, 2000

10. Figueiredo EG, Zabramski JM, Deshmukh P, Crawford NR, Preul MC, Spetzler RF: Anatomical and quantitative description of the transcavernous approach to interpeduncular and prepontine cisterns. Technical note. J Neurosurg 104:957964, 2006

11. Goehre F, Kamiyama H, Noda K, Ota N, Tsuboi T, Miyata S, et al: Technical description of the medial and lateral anterior temporal approach for the treatment of complex proximal posterior cerebral artery aneurysms. World Neurosurg 86:490-496, 2016

12. Goehre F, Lehecka M, Jahromi BR, Lehto H, Kivisaari R, Hijazy F, et al: Subtemporal approach to posterior cerebral artery aneurysms. World Neurosurg 83:842-851, 2015

13. Gross BA, Batjer HH, Awad IA, Bendok BR, Du R: Brainstem cavernous malformations: 1390 surgical cases from the literature. World Neurosurg 80:89-93, 2013

14. Hamlat A, Morandi X, Riffaud L, Carsin-Nicol B, Haegelen $\mathrm{C}$, Helal H, et al: Transtemporal-transchoroidal approach and its transamygdala extension to the posterior chiasmatic cistern and diencephalo-mesencephalic lesions. Acta Neurochir (Wien) 150:317-328, 2008

15. Hebb MO, Spetzler RF: Lateral transpeduncular approach to intrinsic lesions of the rostral pons. Neurosurgery 66 (3 Suppl Operative):26-29, 2010

16. Hu P, Liang J, Bao Y, Li M, Ling F: The pterional transsylvian transtentorial approach to ventrolateral pontine cavernomas: indications and techniques. World Neurosurg 82:1276-1282, 2014

17. Ikeda K, Shoin K, Mohri M, Kijima T, Someya S, Yamashita J: Surgical indications and microsurgical anatomy of the transchoroidal fissure approach for lesions in and around the ambient cistern. Neurosurgery 50:1114-1120, 2002

18. Kalani MY, Yagmurlu K, Martirosyan NL, Cavalcanti DD, Spetzler RF: Approach selection for intrinsic brainstem pathologies. J Neurosurg 125:1596-1607, 2016

19. Kim YD, Elhadi AM, Mendes GA, Maramreddy N, Agrawal A, Kalb S, et al: Quantitative study of the opticocarotid and carotid-oculomotor windows for the interpeduncular fossa, before and after internal carotid artery mobilization and posterior communicating division. Neurosurgery 11 (Suppl 2):162-180, 2015

20. Lawton MT: Seven Aneurysms: Tenets and Techniques for Clipping. New York: Thieme, 2010

21. Lawton MT, Abla AA, Rutledge WC, Benet A, Zador Z, Rayz $\mathrm{V}$, et al: Bypass surgery for the treatment of dolichoectatic basilar trunk aneurysms: a work in progress. Neurosurgery 79:83-99, 2016

22. MacDonald JD, Antonelli P, Day AL: The anterior subtemporal, medial transpetrosal approach to the upper basilar artery and ponto-mesencephalic junction. Neurosurgery 43:84-89, 1998

23. Ota N, Goehre F, Miyazaki T, Kinoshita Y, Matsukawa H, Yanagisawa T, et al: Bypass revascularization applied to the posterior cerebral artery. World Neurosurg 96:460-472, 2016 
24. Post N, Russell SM, Jafar JJ: Role of uncal resection in optimizing transsylvian access to the basilar apex: cadaveric investigation and preliminary clinical experience in eight patients. Neurosurgery 56 (2 Suppl):274-280, 2005

25. Rhoton AL Jr: The posterior fossa cisterns. Neurosurgery 47 (3 Suppl):S287-S297, 2000

26. Rhoton AL Jr: The supratentorial arteries. Neurosurgery 51 (4 Suppl):S53-S120, 2002

27. Rhoton AL Jr: Tentorial incisura. Neurosurgery 47 (3 Suppl):S131-S153, 2000

28. Sabatino G, Rigante L, Marchese E, Albanese A, Esposito G, Capone G, et al: Anterior subtemporal approach for posterolateral brainstem cavernomas: report of ten cases. Acta Neurochir (Wien) 154:2009-2016, 2012

29. Sanai N, Zador Z, Lawton MT: Bypass surgery for complex brain aneurysms: an assessment of intracranial-intracranial bypass. Neurosurgery 65:670-683, 2009

30. Sano K: Temporo-polar approach to aneurysms of the basilar artery at and around the distal bifurcation: technical note. Neurol Res 2:361-367, 1980

31. Seoane E, Tedeschi H, de Oliveira E, Wen HT, Rhoton AL Jr: The pretemporal transcavernous approach to the interpeduncular and prepontine cisterns: microsurgical anatomy and technique application. Neurosurgery 46:891-899, 2000

32. Seoane ER, Tedeschi H, de Oliveira E, Siqueira MG, Calderón GA, Rhoton AL Jr: Management strategies for posterior cerebral artery aneurysms: a proposed new surgical classification. Acta Neurochir (Wien) 139:325-331, 1997

33. Steňo J, Bízik I, Steňová J, Timárová G: Subtemporal transtentorial resection of cavernous malformations involving the pyramidal tract in the upper pons and mesencephalon. Acta Neurochir (Wien) 153:1955-1962, 2011

34. Takami T, Ohata K, Nishikawa M, Goto T, Terakawa Y, Inoue Y, et al: Transposition of the oculomotor nerve for resection of a midbrain cavernoma. Technical note. J Neurosurg 98:913-916, 2003

35. Takeuchi S, Tanikawa R, Tsuboi T, Noda K, Oda J, Miyata S, et al: Superficial temporal artery to proximal posterior cerebral artery bypass through the anterior temporal approach. Surg Neurol Int 6:95, 2015

36. Tayebi Meybodi A, Lawton MT, Griswold D, Mokhtari P, Payman A, Tabani H, et al: Revascularization of the upper posterior circulation with the anterior temporal artery: an anatomical feasibility study. J Neurosurg [epub ahead of print September 22, 2017; DOI: 10.3171/2017.3.JNS162865]

37. Terasaka S, Sawamura Y, Kamiyama H, Fukushima T: Surgical approaches for the treatment of aneurysms on the P2 segment of the posterior cerebral artery. Neurosurgery 47:359-366, 2000

38. Ulm AJ, Tanriover N, Kawashima M, Campero A, Bova FJ, Rhoton A Jr: Microsurgical approaches to the perimesencephalic cisterns and related segments of the posterior cerebral artery: comparison using a novel application of image guidance. Neurosurgery 54:1313-1328, 2004

39. Wang WX, Xu BN, Wang FY, Wu C, Sun ZH: Microsurgical management of posterior cerebral artery aneurysms: a report of thirty cases in modern era. Br J Neurosurg 29:406-412, 2015

40. Wu A, Chang SW, Deshmukh P, Spetzler RF, Preul MC: Through the choroidal fissure: a quantitative anatomic comparison of 2 incisions and trajectories (transsylvian transchoroidal and lateral transtemporal). Neurosurgery 66 (6 Suppl Operative):221-229, 2010

41. Yagmurlu K, Rhoton AL Jr, Tanriover N, Bennett JA: Threedimensional microsurgical anatomy and the safe entry zones of the brainstem. Neurosurgery 10 (Suppl 4):602-620, 2014

42. Youssef AS, Abdel Aziz KM, Kim EY, Keller JT, Zuccarello M, van Loveren HR: The carotid-oculomotor window in exposure of upper basilar artery aneurysms: a cadaveric morphometric study. Neurosurgery 54:1181-1189, 2004

43. Zador Z, Lu DC, Arnold CM, Lawton MT: Deep bypasses to the distal posterior circulation: anatomical and clinical comparison of pretemporal and subtemporal approaches. Neurosurgery 66:92-101, 2010

44. Zeal AA, Rhoton AL Jr: Microsurgical anatomy of the posterior cerebral artery. J Neurosurg 48:534-559, 1978

45. Zhong J, Li ST, Xu SQ, Wan L: Surgical treatment of ventral mesencephalic cavernoma. Acta Neurochir (Wien) 149:1057-1061, 2007

\section{Disclosures}

The authors report no conflict of interest concerning the materials or methods used in this study or the findings specified in this paper.

\section{Author Contributions}

Conception and design: Lawton, Tayebi Meybodi. Acquisition of data: Tayebi Meybodi, Gandhi. Analysis and interpretation of data: Tayebi Meybodi, Gandhi. Drafting the article: Tayebi Meybodi. Critically revising the article: Tayebi Meybodi, Gandhi, Mascitelli, Bozkurt, Bot, Preul. Reviewed submitted version of manuscript: all authors. Approved the final version of the manuscript on behalf of all authors: Lawton. Statistical analysis: Tayebi Meybodi, Gandhi. Administrative/technical/material support: Lawton, Preul. Study supervision: Lawton.

\section{Supplemental Information Companion Papers}

Mascitelli JR, Gandhi S, Tayebi Meybodi A, Lawton MT: The oculomotor-tentorial triangle. Part 2: a microsurgical workspace for vascular lesions in the crural and ambient cisterns. DOI: 10.3171/2018.2.JNS173141.

\section{Correspondence}

Michael T. Lawton: Barrow Neurological Institute, Phoenix, AZ. michael.lawton@barrowbrainandspine.com. 